\title{
TREATMENT OF DENTINAL HYPERSENSITIVITY USING DESENSITIZING AGENTS PLUS SOFT LASER IRRADIATION. A RANDOMIZED COMPARATIVE CLINICAL TRIAL
}

\author{
Mona M. Ghoneim * and Nayer Aboelsaad **
}

\begin{abstract}
This study aimed to evaluate the efficacy of diode laser combined with two in-office desensitizing agents $8 \%$ Arginine-CaCO3 and $1.23 \mathrm{NaF}$ varnish on reducing dentine hypersensitivity (DH) in periodontitis patients.
\end{abstract}

Materials and Methods: Forty patients having stage I and Stage II periodontitis were selected according to the criteria of AAP (2017) with complaint of (DH) after routine periodontal therapy . Patients were randomly divided into two groups: group1- received $\mathrm{NaF}$ varnish then diode laser application at $1 \mathrm{~W}(\mathrm{PW}),(\mathrm{CW})$ for 30 seconds using $320 \mu$ fiber. Group-2, treated with $8 \%$ Arginine-CaCO3 plus same laser irradiation. Each tooth received three application Dentine hypersensitivity evaluation was by tactile, air-blast, and thermal stimuli and measured using VAS scores. The patient's response was recorded at baseline, one month and 3 month after the application.

Results and conclusions: The results were statistically analyzed, and it was found that $8 \%$ Arginine-CaCO3 plus laser treatment was more effective than $1.23 \% \mathrm{NaF}$-varnish plus laser at time intervals. Sensitivity score differences between the groups were statistically significant at one and three months. The $8 \%$ Arginine-CaCO3 group exhibited statistically significant reduction in dentine hypersensitivity on three stimuli at baseline to one and three months. It was concluded that $8 \%$ Arginine- $\mathrm{CaCO} 3$ plus laser irradiation is more effective than $1.23 \% \mathrm{NaF}$ varnish plus laser irradiation in reduction of patients' pain in periodontitis patients.

KEYWORDS: Dentin hypersensitivity, Diode laser, desensitizing agents

\section{INTRODUCTION}

Dentine hypersensitivity (DH) is a very common dental condition that affects adversely the quality of life of those who suffer from the problem. ${ }^{1}$ The pain is usually sharp and of short duration upon exposure to evaporative, tactile, thermal, chemical, or osmotic stimuli. More importantly, no dental pathology or dental defect exists. ${ }^{2}$

\footnotetext{
* Associate Professor of Restorative Dentistry, Faculty of Dentistry, Alexandria university, Egypt.

** Associate Professor of Periodontology, Faculty of Dentistry, Beirut Arab University, Lebanon
} 
Dentin hypersensitivity is often related to patients with in- correct tooth brushing habits, xerostomia, bulimic patients, inappropriate diet, and other factors . The prevalence of dental hypersensitivity is reported to reach $60-98 \%$ among people with periodontal diseases. Gingival recession is the most prevalent clinical cause of $\mathrm{DH}$, which exposes the root surface as in the loss of enamel/cementum by erosion, abrasion, attrition abfraction. ${ }^{3,4}$ Additionally, dental professionals may contribute to dentine exposure and dentine hypersensitivity by over instrumentation. Denuding of root surfaces is a usual encounter in periodontal treatment, surgical/dental operative procedures, and periodontal disease. ${ }^{5,6}$

The widely accepted theory for DH is the theory of hydrodynamic proposed Brannström et al. in 1967; which advocates the dentinal tubule fluid's movement as a main factor for the stimulation of pulpal receptors. ${ }^{7,8}$ Thus, a decrease in dentin sensitivity should be related to a reduction in the ability of fluid to move within dentinal tubules. ${ }^{9}$ Therefore, sealing of these exposed tubules can decrease the intensity of pain resulting from $\mathrm{DH}$.

Based on the hydrodynamic theory, multiple treatment methods have been suggested for in-office DH therapy, such as desensitizing agents as the application of potassium ion, oxalates, sodium fluoride, iontophoresis, adhesives, and resins. ${ }^{10,11,12}$

Lasers have been recently been introduced as another possibility for in-office treatment of $\mathrm{DH}$ and may open up new horizons in the treatment of dentin hypersensitivity. Laser photobiomodulating action on the dental pulp was investigated by many authors. ${ }^{13,14,15,16}$ The mechanism of action of lasers in DH is not fully understood, although several theories have been suggested. Low-intensity lasers act on the cellular level increasing the deposition of tertiary dentin by the odontoblastic cells while middle-output-power lasers act on the dentinal tubules by reducing or obliterating them. ${ }^{17,18}$ The combined effect of laser irradiation with chemical agents such as sodium fluoride and stannous fluoride have been reported to increase the treatment effectiveness by more than $20 \%$ over that of laser treatment only. 19

Of interest is the fact that the laser irradiation can augment the effect of the desensitizer for a longer duration than when they are used alone. They hypothesized that laser favor the durability of the desensitizer for extended time. Some studies recommend desensitizing agent to remain above tooth surface for one minute before laser irradiation

Accordingly, the aim the aim of the present study was to assess the efficacy of diode laser combination with topical desensitizing agents $8 \%$ Arginine$\mathrm{CaCO} 3$ and $1.23 \mathrm{NaF}$ varnish in the treatment of Dentinal Hypersensitivity (DH) in periodontitis patients. Also, to assess the duration of sensitivity relief up to 3 months.

\section{MATERIAL AND METHODS}

This randomized clinical study was conducted after the approval of the Institutional Review Board (IRB) at Beirut Arab University (BAU_, Lebanon; with code number 2015H-016-D-R-0050. Before intervention, patients were briefed about the study and informed consent was obtained from patients after a thorough explanation of the safety and potential efficacy of desensitizing agents, and the probability of receiving both diode laser applications and $1.23 \% \mathrm{NaF}-$ varnish/or $8 \%$ Arginine-CaCO3.

Forty patients having stage I and Stage II periodontitis were selected according to the criteria of AAP (2017) from the Department of Periodontology, faculty of dentistry -BAU. Their age (range 35-55 years) with a history of DH on at least two tooth were selected.

\section{Inclusion criteria}

Non-smokers, disease free patients with good oral hygiene. With at least two vital teeth with hypersensitivity on facial surfaces to thermal, mechanical, tactile stimuli. They had DH following periodontal therapy. 


\section{Exclusion criteria}

Carious lesions, defective restorations, fractured teeth, prosthesis or any painful pathology restored less than three months; or any restorations into the test area.

Additionally, those who have used any desensitizing agents, antibiotics, or undergone any periodontal surgery in the last 6 months were excluded from the study.

All subjects were given instructions to use soft bristled toothbrush twice daily (morning and evening) for 2 minutes with non-desensitizing toothpastes before the baseline examination and during the trial. Moreover, they were instructed not to use any other desensitizing agents during the study.

The equipment and the materials used in the study (Figure 1-4): diode laser (Sirona) application at $1 \mathrm{~W}(\mathrm{PW})$ continues wave mode $(\mathrm{cW})$ for 30 seconds using $320 \mu$ fiber. $\mathrm{NaF}$ varnish. Each $\mathrm{ml}$ contains NaF I.P $50 \mathrm{mg}$ equivalent to $22.6 \mathrm{mg}$ of fluoride in slow release form, 22,600 ppm of fluoride, and PRG barrier coat varnish contains $8 \%$ Arginine-CaCO3

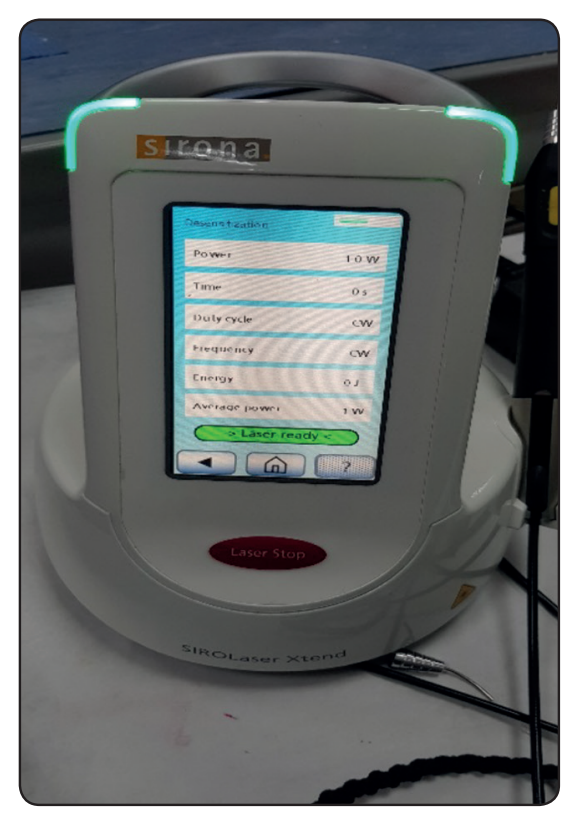

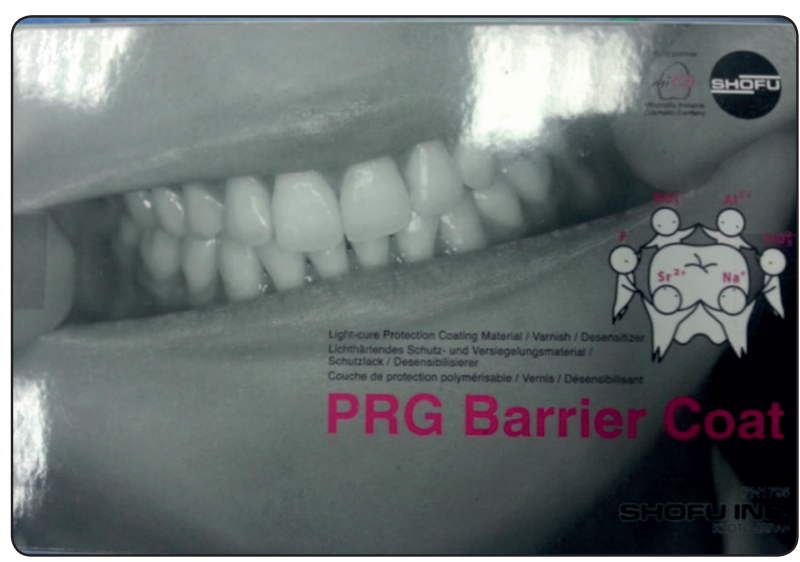

Fig. (2) The desensitizing agent $8 \%$ Arginine-CaCO3 used in this study

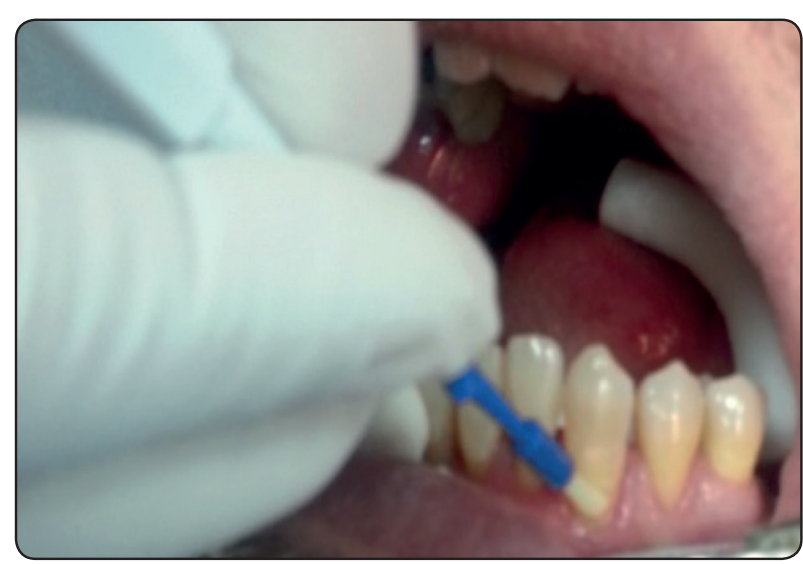

Fig. (3) Topical application of desensitizing agent

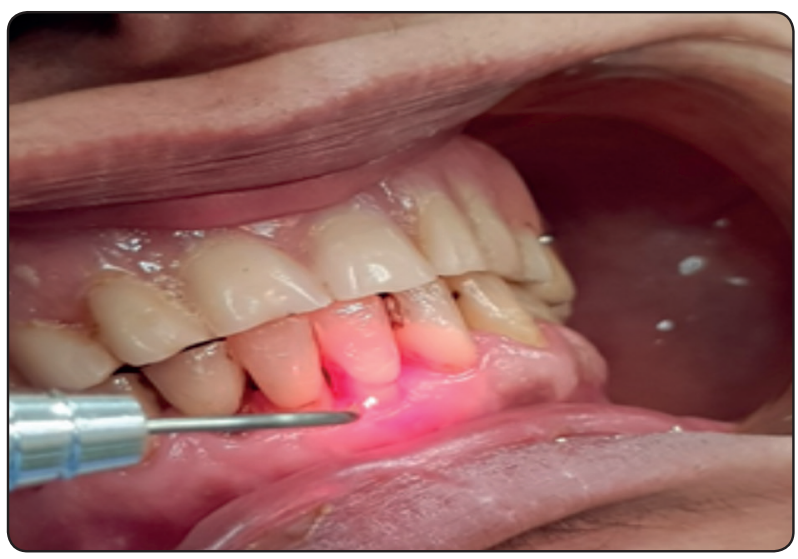

Fig. (4) Diode Laser application on exposed root

Fig. (1) Sirona Laser machine used in this study 


\section{Assessment of hypersensitivity}

\section{Tactile hypersensitivity}

Tactile hypersensitivity was assessed by scratching on the dentinal surface with a sharp-tipped probe and performed by one examiner. The probe tip was placed perpendicular to the evaluable tooth surfaces, just apical to the cemento enamel junction and drawn slowly across the surface in a distal to mesial direction to ensure application of the stimulus across all patent tubules.

\section{Cold water (thermal stimulus)}

$10 \mu \mathrm{l}$ of ice-cold water applied to the exposed dentin surface while neighboring teeth were isolated during testing using cotton rolls. Sensitivity was measured using VAS score. A period of at least 5 minutes was allowed between the two stimuli on each tooth.

\section{Air blast hypersensitivity}

The test teeth were isolated from the adjacent teeth by the placement of red boxing wax. Air was delivered from a standard dental unit air syringe at maximal pressure (45 psi) and at an environmental temperature of 19-24C. The air current was applied for $1 \mathrm{~s}$ at a distance of $1 \mathrm{~cm}$ and perpendicular to the buccal surface of the tooth. The subjects scored pain intensity by placing a mark on a $10 \mathrm{~cm}$-long line on a Visual Analogue Scale (VAS) that was labeled from $(0=$ no pain to $10=$ extreme, unbearable pain). Patients were instructed to point to the VAS. $[9,10]$. Patients were informed before testing about the different score levels. Each group was evaluated at baseline; 1 , and 3 months post application

\section{Statistical methods}

Within treatment, comparisons of the baseline versus the follow-up values were performed using paired t-tests. Comparisons between treatment groups at post-baseline time-points were performed using analyses of covariance (ANCOVA), in which the baseline scores were employed as a co-variable. All comparative statistical tests were two-sided, and employed a level of significance of 0.05 .

\section{RESULTS}

During the study, there were no adverse effects on the soft or hard tissues, which were observed by the examiner or reported by the subjects when questioned. The lateral and central incisors were the most affected tooth in both groups of this study, followed by canines and premolars, while molars were the least affected.

Throughout the study, plaque accumulation was minimal and gingival health was excellent in most of the subjects. There were no statistically significant differences on clinical parameters between groups at first visit $(\mathrm{p}>0.05)$

The VAS scores for three stimuli of all two groups were not statistically different from each other at baseline $(p>0.05)$. (Table 1$)$

The laser plus $8 \%$ Arginine-CaCO3 was found to be better in reducing VAS score for air-blast stimuli tactile stimuli and thermal stimuli compared to the laser plus NaF-gel group. The changes of airblast stimuli and thermal stimuli were highly significant in the laser plus $8 \%$ Arginine- $\mathrm{CaCO} 3$ group at 1 month than laser plus NaF-gel alone group $(\mathrm{p}<0.001)$. The laser plus $8 \%$ Arginine-CaCO3 group was more effective for tactile stimuli than laser NaF- arnish group at 1 month, however the differences between groups was not statistically significant ( $>00.05)$. (Table 1)

The laser plus8 \% Arginine-CaCO3 group: The differences of VAS score for air-blast stimuli, tactile stimuli and thermal stimuli were significant at baseline to 1 month $(\mathrm{p}<0.05)$. There was greater reduction insensitivity score for thermal stimuli at 1 month, following air-blast and tactile stimuli respectively. The VAS score was statistically significant at time interval during experiment for three stimuli. 
TABLE (1) Sensitivity Scores to Three Stimuli for Both Treatment Group at All Time Points.

\begin{tabular}{|c|c|c|c|c|c|c|c|c|c|c|}
\hline & \multicolumn{3}{|c|}{ Thermal } & \multicolumn{3}{c|}{ Tactile } & \multicolumn{3}{c}{ Air Blast } \\
\cline { 2 - 11 } & Baseline & 1 month & 3 months & Baseline & 1 month & 3 months & Baseline & 1 month & 3 months \\
\hline $\begin{array}{c}\text { Laser plus 8 \% } \\
\text { Arginine-CaCO3 }\end{array}$ & $6.56 \pm 0.64 \mathrm{a}, \mathrm{b}$ & $4.24 \pm 0.5$ & $3.24 \pm 0.6 *$ & $5.79 \pm 0.6$ & $4.72 \pm 0.8$ & $3.94 \pm 0.6$ & $5.2 \pm 0.7$ a,b & $3.4 \pm 0.7$ & $3.54 \pm 0.71 *$ \\
\hline Laser plus NaF & $6.26 \pm 0.7$ & $5.5 \pm 0.4$ & $4.3 \pm 0.9 *$ & $5.75 \pm 0.7$ & $5.74 \pm 0.6$ & $4.44 \pm 0.7$ & $5.10 \pm 0.3$ & $4.4 \pm 0.5$ & $4.25 \pm 0.8^{*}$ \\
\hline
\end{tabular}

a "Data are expressed as mean and standard deviation".

b Difference within group according to stimulus was statistically significant $(p<0.05)$.

*Statistically significant at p value $<0.05$

The laser plus $\mathrm{NaF}$ varnish group :The changes in VAS score of air-blast stimuli, tactile stimuli and thermal stimuli at 1 month were decreased to compare with baseline, decreasing was not statistically significant $(\mathrm{p}>0.05)$. At 3 month, the VAS scores for air-blast, tactile and thermal stimuli were lower than baseline. These differences were statistically significant.

\section{DISCUSSION}

Dentine hypersensitivity usually has multifactorial etiology and generally, more than one factor is found associated and active in this painful sign; therefore, more than one treatment method should be associated to desensitize the dentine to satisfactory levels. The results of this study provide important insight into possible mechanism(s) of spontaneous disappearance of hypersensitive dentin.

Conventional therapies for the treatment of DH comprehend the topical use of desensitizing agents, either professionally or at home such as protein precipitants, tubule-occluding agents, tubule sealants [18-20], and, recently, lasers. The features of an ideal desensitizing technique/material as described by Lutin ND et al, are; it should be easy to apply, non-irritating to the pulp tissue, painless, consistent and effective in long term, quick acting and produce no discoloration. Moreover, modification of dental hard tissue by laser irradiation is becoming increasingly popular in dentistry.
We used the VAS in this study to evaluate dentin hypersensitivity, previously, several investigators have been demonstrated the validity and reliability of the VAS. The VAS also appears to be more sensitive in discriminating between various treatments and changes in pain intensity ${ }^{(30)}$

Several authors studied the effectiveness of the diode laser and reported from $60 \%$ to $85 \%$ improvement in teeth treated with laser compared to $20 \%$ average of the control nonlased group. ${ }^{(18)}$

The laser power parameter used in this study was $1 \mathrm{~W} / \mathrm{CW}$ which was not agreeable by previous studies done by (Liu et al, 2013) (Suri et al, 2016) who used $2 \mathrm{~W} / \mathrm{CW}$. There is no universal agreement in laser parameters usage in different dental laser application. Therefore there is urgent need of conducting adequately powered and well-structured randomized control clinical trials to overcome the heterogeneity present in the literature .

Diode laser leads to increase in mitochondrial ATP through bio-stimulation, increases pain threshold of free nerve ending, provide analgesic effect by increase in endorphins. It also inhibits cyclooxygenase enzyme which causes conversion of arachidonic acid into prostaglandin which in turn increases the pain transmission by glutamate or substance P. There is also formation of secondary dentin by odontoblast due to bio-stimulation ${ }^{[23-25]}$. 
In addition to diode lasers, Erbium, $\mathrm{CO} 2$, and Nd:YAG, frequently are used to treat dentinal hypersensitivity. Studies mainly focus on obliteration of the dentinal tubules but disregard the laser's additional biostimulatory effect. PBM will not modify the dentinal tubules but will produce an effect in the odontoblastic layer, stimulate secondary dentin formation, and simultaneously reduce inflammation. In combination with traditional desensitizing agents, PBM is a valuable treatment modality Wakabayashi and Matsumoto showed that use of a low-level diode laser was effective in 61 of 66 cases. The combined use of the GaAlAs laser (830 nm wavelength) with fluoridation enhances treatment effectiveness by more than $20 \%$ over that of laser treatment only. In an in vitro study, most dentinal tubule orifices were occluded after treatment by Nd:YAG laser irradiation followed by topical sodium fluoride ${ }^{(21-23)}$. Previous rsearch used dicalcium phosphate bioglass in combination with $\mathrm{Nd}$ :YAG laser in treatment of $\mathrm{DH}$. According to their study, this combination sealed dentinal tubules to a depth of $10 \mathrm{~mm}{ }^{(27)}$.

Several studies showed that low-level laser treatment promoted significantly better results, establishing an irradiation protocol of three sessions with an interval of $72 \mathrm{~h}$ between them. Because these studies used infrared low-level lasers, Ladalardo et al. studied the influence of different wavelengths on pain reduction and found that the $660-\mathrm{nm}$ red diode was more effective than the 830-nm infrared diode laser. Marsílio et al. observed positive clinical results with use of low-level laser wavelengths in the red spectrum, with pain reduction rates of $86.53 \%$ and $88.88 \%$ for 3 and $5 \mathrm{~J} / \mathrm{cm} 2$, respectively. It was reported that this same wavelength with the fluoride varnish frequently used in the treatment of dentinal hypersensitivity and obtained improved results with LPT. The more sclerotic the pulp chamber, the higher the energies needed; $4 \mathrm{~J}$ to $10 \mathrm{~J}$ is used. As with surgical lasers, patient feedback determines when dentinal sensitivity is decreased or eliminated.

It is probable that the better performance of combined treatment was due to the higher desensitizing agent adhesion to the dentinal tubules when combined with laser energy. It can be hypothesize that the laser-induced superficial melting permits to keep longer the tubules occlusion by these agents emphasizing the reduction of $\mathrm{DH}-$ related pain.

\section{CONCLUSION}

According to these results, the diode laser showed a very high capability to improve immediately the DH-related pain, even better in combination with $8 \%$ Arginine-CaCO3 than with $\mathrm{NaF}$ varnish. These results have to be confirmed by greater samples of patients and by longer follow-up periods (e.g.9 and 12 months) to confirm or not the longlasting action of the combined laser and desensitizing agents therapy.

\section{REFERENCES}

1. Matsumoto K, Kimura Y. Laser therapy of dentin hypersensitivity. J Oral Laser Appl. 2007;7:07-25.

2. Machado AC, Viana ÍEL, Farias-Neto AM, Braga MM, Is photobiomodulation (PBM) effective for the treatment of dentin hypersensitivity? A systematic review. Lasers Med Sci. 2018 May;33(4):745-753.

3. Holland GR, Närhi MN, Addy M, Gangarosa L, Orchardson R (1997). Guidelines for the design and conduct of clinical trials on dentine hypersensitivity. J Clin Periodontol 24:808-813.

4. Mitra Askari, Reza Yazdani. Comparison of two desensitizing agents for decreasing dentin hypersensitivity following periodontal surgeries: a randomized clinical trial. Quint Int, volume 50 • number 4 • April 2019

5. Zero DT, Lussi A. Erosion--chemical and biological factors of importance to the dental practitioner. Int Dent $\mathrm{J}$ 2005; 55: 285-290.

6. Bartold PM. Dentinal hypersensitivity: a review. Aust Dent J 2006;51:212-218.

7. Davari AR., Ataei E., Assarzadeh H. Dentin Hypersensitivity: Etiology, Diagnosis and Treatment; A Literature Review. J Dent Shiraz Univ Med Sci, Sept. 2013; 14(3): 136-145.

8. Brännström M, Åström A. The hydrodynamics of the dentine; its possible relationship to dentinal pain. Int Dent $\mathrm{J}$ 1972;22:219- 227. 
9. Greenhill, J.D. and Pashley D.H.: The Effect of Desensitizing Agents on the Hydraulic Conductance of Human Dentin ,invitro,JDentRes60:686-698,1981.

10. Lan WH, Liu HC (1996). Treatment of dentin hypersensitivity by Nd:YAG laser. J Clin Laser Med Surg 14:89-92.

11. Lan WH, Liu HC, Lin CP (1999). The combined occluding effect of sodium fluoride varnish and Nd:YAG laser irradiation on human dentinal tubules. J Endod 25:424-426.

12. Porto IC, Andrade AK, Montes MA (2009). Diagnosis and treatment of dental hypersensitivity. J Oral Sci 51:323-332.

13. Sgolastra F., A. Petrucci, M. Severino, R. Gatto, and A. Monaco. Lasers for the Treatment of Dentin Hypersensitivity: A Meta-analysis. J Dent Res 92(6):492-499, 2013

14. Thomas George V, T Aby Mathew, Nebu George, Saumya John,,Sneha M Prakash, M S Vaseem. Efficacy of Diode Laser in the Management of Dentin Hypersensitivity Following . Periodontal Surgery Journal of International Oral Health 2016; 8(1):103-108

15. Renton-Harper P, Midda M. Nd:YAG laser treatment of dentinal hypersensitivity. Brit Dent J 1992;172:13-16.

16. Schwarz F, Arweiler N, Georg T, Reich E. Desensitizing effects of an Er:YAG laser on hypersensitive dentin. J Clin Periodontol 2002;29:211-215.

17. Ladalardo TC, Pinheiro A, Campos RA, Brugnera Júnior A, Zanin F, Albernaz PL, et al. (2004). Laser therapy in the treatment of dentine hypersensitivity. Braz Dent J 15:144-150.

18. Sgolastra F, Petrucci A, Gatto R, Monaco A (2011). Effectiveness of laser in dentinal hypersensitivity treatment: a systematic review. J Endod 37:297-303.

19. Liu H-C, Lan W-H. The combined effectiveness of the semiconductor laser with Duraphat in the treatment of dentin hypersensitivity. J Clin Laser Med Surg 1994;12:315-319.

20. 20. Papapanou PN, Sanz M, Buduneli N, Dietrich T, Feres M, Fine DH, et al,. Periodontitis: Consensus report of workgroup 2 of the 2017 World Workshop on the Classification of Periodontal and Peri-Implant Diseases and Conditions. J Periodontol. 2018 Jun;89 Suppl 1: S173-S182.

21. Liu Y, Gao J, Gao Y, Xu S, Zhan X, Wu B. In vitro study of dentin hypersensitivity treated by $980-\mathrm{nm}$ diode laser. J Lasers Med Sci 2013;4:111-9
22. Suri I, Singh P, Shakir QJ, Shetty A, Bapat R, Thakur R. A . comparative evaluation to assess the efficacy of 5\% sodium fluoride varnish and diode laser and their combined application in the treatment of dentin hypersensitivity. J Indian Soc Periodontol 2016;20:307-14.

23. Praveen R, Thakur S, Kirthiga M, Narmatha M. Comparative evaluation of a low-level laser and topical desensitizing agent for treating dentinal hypersensitivity: A randomized controlled trial. J Conserv Dent. 2018 SepOct;21(5):495-499

24. Lopes AO, Eduardo Cde P, Aranha AC. Clinical evaluation of low-power laser and a desensitizing agent on dentin hypersensitivity. Lasers Med Sci. 2015 Feb;30(2):823-9.

25. Gojkov-Vukelic M, Hadzic S, Zukanovic A, Pasic E, Pavlic V. Application of Diode Laser in the Treatment of Dentine Hypersensitivity. Med Arch. 2016 Dec;70(6):466-469.

26. El Mobadder M, Namour A, Namour M, Dib W, El Mobadder W, et al,. Dentinal Hypersensitivity Treatment Using Diode Laser 980 nm: In Vivo Study. Dent J (Basel). 2019 Jan 9;7(1). pii: E5.

27. Tunar OL, Gürsoy H, Çakar G, Kuru B, Ipci SD, Yılmaz S . Evaluation of the effects of Er:YAG laser and desensitizing paste containing $8 \%$ arginine and calcium carbonate, and their combinations on human dentine tubules: a scanning electron microscopic analysis. Photomed Laser Surg. 2014 Oct; 32(10):540-5.

28. Wakabayashi H. and. Matsumoto K, "Study on the treatment of hypersensitive dentine by laser," Journal of Conservative Dentistry, vol. 28, pp. 1366-1371, 1985

29. Kapferer I, Pflug C, Kisielewsky I, Giesinger J, Beier US, Dumfahrt H. Instant dentin hypersensitivity relief of a single topical application of an in-office desensitizing paste containing $8 \%$ arginine and calcium carbonate: A splitmouth, randomized-controlled study .Acta Odontologica Scandinavica, 2013; 71: 994-999

30. Soares ML, Porciúncula GB, Lucena MI, Gueiros LA, Leão JC, Carvalho AA. Efficacy of Nd:YAG and GaAlAs lasers in comparison to $2 \% \mathrm{fl}$ uoride gel for the treatment of dentinal hypersensitivity. Gen Dent. 2016 Nov-Dec; 64(6):66-70.

31. Brahmbhatt N, Bhavsar N, Sahayata V, Acharya A, Kshatriya $\mathrm{P}$. A double blind controlled trial comparing three treatment modalities for dentin hypersensitivity. Med Oral Patol Oral Cir Bucal. 2012;17:e483-90. 